\title{
Impulso de la Red de Divulgación de Ciencia, Tecnología e Innovación como organismo promotor de la sociedad del conocimiento en San Luis Potosí, México
}

Promotion of the Red de Divulgación de Ciencia, Tecnología e Innovación (Science, Technology and Innovation Dissemination Network) as a promoter of the society of knowledge in San Luis Potosí, Mexico

\section{ISSN 2071-8748 \\ E-ISSN 2218-3345

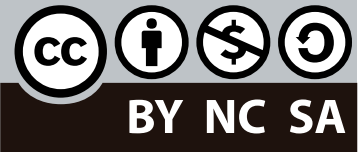

DOI: https://doi.org/10.5377/entorno.v0i68.8453

URI: http://hdl.handle.net/11298/1133
Edgar Jiménez-Félix https://orcid.org/0000-0002-9380-9495 Alumno de Doctorado en Educación, Universidad Tangamanga Consejo Potosino de Ciencia y Tecnología, Gobierno de San Luis Potosí 2015-2021 ejimenez@copocyt.gob.mx

Recibido: 26 de junio 2019 Aprobado: 10 de septiembre 2019

\section{Resumen}

La Red de Divulgación de Ciencia, Tecnología e Innovación (Rediciti) fue una estrategia que impulsó el Consejo Potosino de Ciencia y Tecnología (Copocyt) en el año 2010 con el objetivo de captar, profesionalizar e impulsar las acciones de divulgación científica en el estado. Esta comunidad de divulgadores ha permitido la atención de población de alta y muy alta marginación, permitiendo identificar necesidades educativas, sociales y tecnológicas en las cuatro regiones del estado. El modelo conceptual que se utilizó para el impulso de las actividades de la Red fue el de Jorge Padilla y Lourdes Patiño de la Sociedad

\section{Abstract}

The Red de Divulgación de Ciencia, Tecnología e Innovación (Science, Technology and Innovation, Rediciti, given its Spanish acronym) was a strategy promoted by the Consejo Potosino de Ciencia y Tecnología (the Potosino Science and Technology Council, Copocyt, given its Spanish acronym) during 2010, with the objective to recruit, professionalize and promote all actions directed towards scientific dissemination in the state. Said community of disseminators has given room for providing attention to populations with a high, and very high marginalization, allowing to identify the educational, 
Mexicana para la Divulgación de la Ciencia y la Técnica A.C. (Somedicyt), dando como resultado actividades de alto impacto.

\section{Palabras clave}

Divulgación científica - San Luis Potosí, México. Ciencia y tecnología - San Luis Potosí, México. Investigación científica - San Luis Potosí, México. Investigación socia San Luis Potosí, México. social and technological needs in the four regions of the state. The conceptual model used for the promotion of the activities conducted by the network was that of Jorge Padilla and Lourdes Patiño from the Sociedad Mexicana para la Divulgación de la Ciencia y la Técnica, A.C. (Mexican Society for the Dissemination of Science and Techniques, Somedicyt, given its Spanish acronym); high impact activities were a result.

\section{Keywords}

Scientific dissemination-San Luis Potosí, Mexico. Science and technology - San Luis Potosí, Mexico. Scientific research - San Luis Potosí, Mexico. Social research - San Luis Potosí, Mexico.

\section{Introducción}

El progreso de toda sociedad en un mundo que cambia de forma permanente y en el que la población deba de adaptarse y resolver los problema de la cotidianeidad, dependerá de la capacidad de apropiarse del conocimiento humanístico, científico, tecnológico y de innovación que le permita tener acceso a información, tener juicio crítico acerca de ella y así generar nuevos conocimientos.

Ante la inminente evolución del concepto sociedad del conocimiento, se tomará como definición: sociedad que considera cambios en las áreas tecnológicas y económicas estrechamente relacionadas con las tecnologías de la información y comunicación (TIC), en el ámbito de planificación de la educación y formación, de la organización y del trabajo (Krüger, 2006).

Bajo la definición anterior, la divulgación de ciencia, tecnología e innovación (CTI) ha pasado por una transformación en el contexto mundial, es decir, la necesidad de acercar a la población el conocimiento de CTI, tanto en las zonas urbanas como en locaciones remotas con población en condiciones de vulnerabilidad o grupos subrepresentados, demanda estrategias en el uso de las TIC así como en el desarrollo de las habilidades y competencias de quienes transmiten el conocimiento, con el objetivo de convertirse en una sociedad del conocimiento.

Según la Organización de las Naciones Unidas para la Educación, la Ciencia y la Cultura (Unesco, por sus siglas inglés), el intercambio de conocimiento e información tiene un gran impacto para transformar las economías y las sociedades, aumentando el acceso, la preservación y el intercambio en todos sus ámbitos de competencia.

Educación de calidad para todos, acceso universal al conocimiento y a la información, el respeto a la diversidad cultural y lingüística, y la libertad de expresión, se consideran los cuatro pilares fundamentales en los que deben estar basadas las sociedades del conocimiento, de acuerdo con la Unesco. ${ }^{1}$

Conforme a estos lineamientos internacionales, que después derivaron, en México, en elementos considerados en el Plan Nacional de Desarrollo² (PND) 2013-2018 y en el Plan Estatal de Desarrollo ${ }^{3}$ (PED), tanto 2009-2015 como 2015-2021, el Copocyt, a partir de 2010, impulsó la Rediciti con el objetivo de crear una comunidad en la que se tuviera la participación

1 "Construir sociedad del conocimiento" (https://es.unesco.org/themes/construir-sociedades-del-conocimiento)

2 Plan Nacional de Desarrollo 2013-2018 (https://www.dof.gob.mx/nota_detalle_popup.php?codigo=5299465)

3 Plan Estatal de Desarrollo 2015-2021 (http://www.slp.gob.mx/plan2015-2021/) 
de personas formadas, tanto de manera profesional como empírica, en la realización de actividades relacionadas con la divulgación de la CTI con el fin de orientar las actividades a los lineamientos establecidos en la transición hacia la sociedad del conocimiento.

Sin embargo, el camino ha sido complicado ante prácticas acuñadas durante años en el marco de la divulgación de las ciencias, siendo que la principal actividad por realizar eran talleres expositivos de forma presencial, los cuales no habían cambiado su metodología en más de quince años; no existía capacitación ni programas de profesionalización de divulgadores y la participación de las ciencias sociales era prácticamente nula, a diferencia de áreas de ciencias exactas o naturales.

Bajo este panorama, la definición de estrategias y de conceptos fundamentales que orientaran la política pública para el estado de San Luis Potosí sería un proceso integral entre sensibilización de la actividad de la divulgación y una etapa de desaprendizaje, para la eliminación de prácticas no evaluadas ni sistematizadas para validar su impacto social y así lograr la apropiación social del conocimiento.

\section{Método}

De acuerdo con información estadística dada a conocer por el Consejo Nacional de Población (Conapo), San Luis Potosí es la séptima entidad más marginada de la República
Mexicana, solamente detrás de estados como Guerrero, Chiapas, Oaxaca, Veracruz, Puebla e Hidalgo.

En el estudio se refleja que en la entidad potosina habitan 2. 85.518 de personas, de las cuales el 7,96 \% son analfabetas, además el $46,70 \%$ de la población en el estado sobrevive con dos salarios mínimos o menos.

Las cifras muestran que el $23,18 \%$ de la población que cuenta con 15 años de edad o más carece de estudios de primaria. Además, los datos se refieren a condiciones de pobreza que generan marginación social, como lo indica el casi $4 \%$ de la población que no cuenta con los servicios de drenaje, servicios sanitarios ni con energía eléctrica. El $34,43 \%$ de las familias en todo el estado (casi 4 de cada 10 hogares) presentan algún grado de hacinamiento.

Un punto importante que se debe destacar, por lo que San Luis Potosí ocupa este séptimo lugar de los estados más marginados del país radica en que de acuerdo al estudio de Conapo, poco menos de la mitad de la población $(40,08 \%)$ vive en localidades aisladas de menos de 5.000 habitantes, circunstancia que limita su desarrollo y sus oportunidades.

Los datos anteriormente expuestos arrojan un índice de marginación para San Luis Potosí de 0.56416, es decir, es el cuarto estado con una categoría de Alto (tabla 1) dentro de dicho índice. Mismo que contrasta con estados vecinos como Nuevo León (-1.38323) o Coahuila (-1.14000), ambos con una categoría de muy bajo índice de marginación.

Tabla 1. Índice de marginación por entidad federativa en México ${ }^{4}$

\begin{tabular}{|l|c|c|c|c|c|}
\hline \multicolumn{1}{|c|}{ Entidad federativa } & $\begin{array}{c}\text { Población } \\
\text { total }\end{array}$ & $\begin{array}{c}\text { Índice de } \\
\text { marginación }\end{array}$ & $\begin{array}{c}\text { Grado de } \\
\text { marginación }\end{array}$ & $\begin{array}{c}\text { Índice escala } \\
0 \text { a } 100\end{array}$ & $\begin{array}{c}\text { Lugar que ocupa en } \\
\text { el contexto nacional }\end{array}$ \\
\hline Guerrero & 3.388 .768 & 2.53246 & Muy alto & 88.72 & 1 \\
\hline Chiapas & 4.796 .580 & 2.31767 & Muy alto & 84.14 & 2 \\
\hline Oaxaca & 3.801 .962 & 2.14624 & Muy alto & 80.48 & 3 \\
\hline Veracruz & 7.643 .194 & 1.07546 & Alto & 57.63 & 4 \\
\hline Puebla & 5.779 .829 & 0.71224 & Alto & 49.88 & 5 \\
\hline Hidalgo & 2.665 .018 & 0.66143 & Alto & 48.79 & 6 \\
\hline San Luis Potosí & $\mathbf{2 . 5 8 . 5 5 1 8}$ & $\mathbf{0 . 5 6 4 1 6}$ & Alto & $\mathbf{4 6 . 7 2}$ & $\mathbf{7}$ \\
\hline
\end{tabular}

4 Estimaciones del Conapo con base en Inegi, Censo de Población y Vivienda 2010. (http://www.conapo.gob.mx/work/models/CONAPO/Marginacion/Datos Abiertos/Entidad_Federativa/01_Entidad_Federativa/Mapa_de_grado_de_marginacion_por_entidad_federariva_2015.rar) 
El PND, presentado por la Presidencia de la República 20122018, en su eje III, México con Educación de Calidad, subeje III.2. "Plan de acción: articular la educación, la ciencia y el desarrollo tecnológico para lograr una sociedad más justa y próspera", establece que se deberá de hacer del desarrollo científico, tecnológico y la innovación pilar para el progreso económico y social sostenible; y para ello se requiere una sólida vinculación entre escuelas, universidades, centros de investigación (Cl) y con el sector privado.

Si continuamos en la jerarquía de las políticas públicas establecidas en el marco estatal, se encuentra que, como parte del PED 2015-2021, se realizó el "Diagnóstico del sector de ciencia, tecnología e innovación del estado de San Luis Potosí", el cual establece como estrategia dentro de las metas nacionales 2014-2018 en CTI el contribuir a la transferencia y al aprovechamiento del conocimiento, vinculando a las IES y $\mathrm{Cl}$ con los sectores público, social y privado.

El director nacional del Consejo Nacional de Ciencia y Tecnología (Conacyt) durante el periodo 2012-2018, el Dr. Enrique Cabrero Mendoza, en una ponencia con universitarios, el 4 de octubre de 2017 en San Luis Potosí ${ }^{5}$, explicó a los jóvenes "cómo es que nuestro país debe pensar en convertirse hacia la sociedad del conocimiento". Señaló que lo que hacen otros países es generar conocimiento a través de laboratorios y equipamiento, desde hace ya varios siglos.

El Dr. Cabrero aseguró cómo el conocimiento científico y tecnológico puede transformarse en algo útil económicamente, porque es un tema crucial para las empresas y el gobierno. Destacó que la innovación es un convertidor del conocimiento en donde hay empresarios y gente calificada.

En esta sociedad del conocimiento, hay que estar pendiente de aspectos como: el capital humano, la infraestructura de información y comunicación adecuada, el sistema de innovación y desarrollar toda una cultura de innovación eficaz.
Indicó que todo esto se da para satisfacer las necesidades de la sociedad, su bienestar y sus condiciones de vida. Sobre esto, el funcionario destacó que "México tiene todas las condiciones para ser un país exitoso en la sociedad del conocimiento". Esto permitiría, a nuestro país, convertirse en una sociedad que tome decisiones en materia de inversión en ciencia y tecnología, para avanzar hacia el bienestar social, desarrollando sus capacidades de innovación.

Finalmente, indicó que México se ubica a en el lugar 51 a escala mundial en competitividad; y en el escalón 58 en innovación a escala, indicador que señala que aún estamos lejos de donde deberíamos estar en estas materias.

Por lo anterior, resulta indispensable que la articulación de la triple hélice empresa-academia-gobierno sea funcional en todos sus niveles, con el fin de dar progreso a la transición hacia una sociedad del conocimiento.

La necesidad de acercar el conocimiento a todos los niveles de la sociedad, mediante la divulgación científica, demanda la formación de profesionales de la divulgación, así como la generación de herramientas y espacios para difundir los contenidos científicos a un público diverso, a través de la interacción con los sectores académico, empresarial, gubernamental y social.

La Estrategia Nacional de Fortalecer y Fomentar la Difusión y Divulgación de la Ciencia, la Tecnología y la Innovación (EDDNCTi), ha sido el principal programa de apoyo por parte del Conacyt, que derivó en los resultados obtenidos en materia de divulgación de CTI desde el 2012 y hasta 2019 en San Luis Potosí.

\section{La Rediciti}

Tomando como base es estatus de marginación existente en el estado, los lineamientos internacionales, federales y estatales, así como la responsabilidad de ley que tiene le Copocyt, este inicia en 2010 la propuesta de la creación de la Rediciti, a partir de la integración de instituciones de

5 "México debe caminar hacia la sociedad del conocimiento": Enrique Cabrero. (http://www.uaslp.mx/Comunicacion-Social/Paginas/Divulgacion/Gaceta/Notas/2017/Octubre/061017/Enrique-Cabrero-conferencia.aspx) 
educación superior (IES), $\mathrm{Cl}$ y, sobre todo, todos aquellos grupos con una trayectoria en divulgación de la CTI, tal como el Taller Infantil de Física Espacial (Tife), uno de los grupos con más tiempo en San Luis Potosí, con aproximadamente 40 años cumplidos en 2019.

La Rediciti inicia con 10 miembros, personas que, principalmente por afición o por tener un puesto institucional, participaban en actividades de divulgación de la CTI.

Para 2012, el Conacyt emite la primera convocatoria de la ENDDCTi en las entidades federativas a través del proyecto "Apropiación Social de la Ciencia, la Tecnología y la Innovación", por lo que, a través del Copocyt, se desarrolló el proyecto del primer diplomado en divulgación de la CTI, liderado por la UASLP, con la intención de agremiar a más interesados y personas que desarrollaban actividades de divulgación por su cuenta y comenzar a fortalecer la Rediciti.

En este contexto, para diciembre de 2012, la Rediciti, gracias a los egresados de dicho diplomado, se incrementó en aproximadamente 30 personas. En 2013 inicia de manera formal con reuniones de trabajo mensuales, e inicia con un ejercicio de planeación estratégica liderado por el Copocyt a fin de definir la visión, la misión y los valores de lo que sería la Red. Para mayo del mismo año, cambia de liderazgo la administración de la Rediciti, lo que permitió dar un impulso mayor a las estrategias implementadas en la Red.
Desde 2013 y hasta 2019, por indicaciones de las direcciones generales del Copocyt (Dr. Enrique Villegas Valladares, 20092015; Dr. José Luis Morán López, 2016-2018; y MAPP. Rosalba Medina Rivera 2018- a la fecha del presente artículo), se consideró no liberar o entregar a entidades del exterior al Copocyt la administración y control de la Red. En primer lugar, ante posibles intenciones de monopolizar por parte de los mismos miembros, y en segundo, porque podrían desvirtuarse y politizarse las acciones, perdiendo de vista el objetivo principal de la Red.

Cabe destacar, que las actividades del sector en materia de divulgación científica y tecnológica están basadas en la formulación deestrategiasque puedanafianzar laapropiación social de la ciencia y la tecnología, y no en actividades que solamente lleguen a impactar en un alfabetismo científico (figura 1), así como en una cultura científica, es decir, con base en el estudio realizado y presentado por la Red Nacional de Consejos y Organismos Estatales de Ciencia y Tecnología (Rednacecyt), Fortalecimiento de la Apropiación Social de la Ciencia y la Tecnología en los estados en 2012, indica que la apropiación social de la ciencia y la tecnología existe cuando la población aplica conocimientos de ciencia y herramientas tecnológicas en situaciones pertinentes de la vida cotidiana, así como también participa en asuntos de ciencia y tecnología, desarrollando el hábito de informarse sobre temas y asuntos de ciencia y tecnología.

\section{Figura 1. Modelo conceptual sobre apropiación social, cultura y alfabetismo científico de Padilla y Patiño [2011] (Somedicyt)}

\begin{tabular}{|c|c|}
\hline $\begin{array}{l}\text { Apropiación social de } \\
\text { la ciencia y la } \\
\text { tecnología }\end{array}$ & $\begin{array}{l}\text { - Aplicación de conocimientos de ciencia y herramientas tecnológicas en } \\
\text { situaciones pertinentes de la vida cotidiana } \\
\text { - Participación social en asuntos de ciencia y tecnología } \\
\text { - Informarse sobre temas y asuntos de ciencia y tecnología }\end{array}$ \\
\hline Cultura cientifica & $\begin{array}{l}\text { - Percepción informada de la ciencia y la tecnología } \\
\text { - Comprensión del papel social de la ciencia y la tecnología } \\
\text { - Comprensión del mundo desde una perspectiva científica } \\
\text { - Aplicación de un pensamiento crítico } \\
\text { - Concepción de la ciencia y la tecnología como parte de la cultura }\end{array}$ \\
\hline Alfabetismo científico & $\begin{array}{l}\text { - Conocimiento y conceptos básicos de ciencia } \\
\text { - Comprensión de los métodos de la ciencia } \\
\text { - Aprendizaje de herramientas tecnológicas pertinentes } \\
\text { - Desarrollo de un pensamiento crítico }\end{array}$ \\
\hline
\end{tabular}


Ejercicio de planeación estratégica de la Red de Divulgación

A partir del modelo conceptual de Padilla y Patiño (2011), en 2013 la Dirección General del Copocyt (Dr. Enrique Villegas Valladares, 2009-2015) lideró el ejercicio de planeación estratégica, en la cual participaron integrantes de la Red; y después de un día de mesas de discusión y la realización del análisis de fortalezas, oportunidades, debilidades y amenazas (Foda), se consolidaron los siguientes pilares estructurales y de conceptualización de la Rediciti:

Visión

Ser una comunidad multidisciplinaria líder a escala nacional e internacional que realiza divulgación de ciencia, tecnología e innovación a fin de contribuir al desarrollo humano a través del fomento de las vocaciones científicas, el apoyo a la educación y la gestión de recursos.

Misión

Es una comunidad que impulsa la cultura y las vocaciones científicas y tecnológicas, a través del desarrollo de estrategias y de la gestión para fomentar la divulgación de la ciencia y tecnología en beneficio de la sociedad.

Análisis Foda de la Rediciti

\section{Fortalezas}

1. Capacidad de gestión

2. Respaldo institucional

3. Actividades ya establecidas

4. Promotores de divulgación

\section{Oportunidades}

1. Apertura de los gobiernos Federal y estatal para la incorporación de la divulgación al modelo educativo.

2. Programas de formación de capital humano en materia de divulgación.

3. Incremento presupuestal a CTI.

4. Fondos de financiamiento nacionales.

5. Infraestructura museográfica en el estado y en el país.
Debilidades

1. Número insuficiente de divulgadores.

2. Falta de recursos.

3. Falta de documentación de las experiencias de divulgación.

4. Falta de formación como gestores de recursos.

5. Falta de vinculación entre académicos y divulgadores.

\section{Amenazas}

1. Percepción distorsionada de la ciencia.

2. Desinterés de los actores de CTI en la divulgación.

3. Poco interés de la sociedad hacia la cultura científica.

4. Falta de reconocimientos y estímulos hacia los divulgadores.

5. Falta de reconocimiento curricular y social.

Objetivos que se deben perseguir:

1. Consolidar la Red de Divulgación a nivel estatal mediante la integración, profesionalización y seguimiento permanente de sus integrantes.

2. Elaborar un plan rector de divulgación para el estado.

3. Diseñar indicadores de evaluación para medir y analizar el desempeño de la Red.

4. Generar mecanismos de auto-sustentabilidad financiera.

5. Realizar actividades de divulgación en ámbitos educativos mediante la creación de talleres innovadores que permitan despertar el interés en los alumnos en la ciencia y la tecnología.

6. Realizar actividades de formación de recursos humanos especializados en la divulgación para expandir el conocimiento científico en todo el estado.

7. Establecer los mecanismos para la gestión de recursos económicos que permitan la divulgación de la ciencia y tecnología en todos los ámbitos.

8. Triplicar el número de divulgadores en el estado.

9. Quintuplicar el presupuesto para divulgación.

10. Atender los 58 municipios del estado de San Luis Potosí.

El ejercicio de planeación estratégica permitió ser la base para el desarrollo de las actividades de carácter estructural y administrativo para la Red, que dio resultado a las siguientes acciones que se describen:

\footnotetext{
6 Copocyt, 2013. Reporte final del programa Apropiación social de la ciencia, tecnología e innovación con énfasis en zonas marginadas del estado de San Luis Potosí 2013 "Ciencia para todos y en todos los rincones".
} 
En $2013^{6}$ se coordinaron 7 delas 12 reuniones del calendario en distintas IES y el Instituto Potosino de Investigación Científica y Tecnológica A.C. (Ipicyt) como sedes. Se abordaron puntos como: seguimiento de acuerdos, eventos en General Motors, Apostólica, seguimiento diplomado de Divulgación de la UASLP que no había entregado reconocimientos, encuentro nacional de divulgación, campings de verano en centros comunitarios, convocatorias Conacyt, Semana Nacional, Jornadas, Presentación de los grupos de divulgación y sus respectivas actividades y trámites para una futura formalización.

Para 2014,7 se coordinaron ocho reuniones y el 1er Encuentro de Divulgadores en el Ipicyt. Los temas que se trabajaron durante dicho año fueron: seguimiento de acuerdos, presentación del Museo Interactivo de Tecnología Avanzada, instalado en la Facultad de Ingeniería de la UASLP, presentación de actividades de divulgación de IES y Cl de la Red, "Día del Niño", actividades para la Semana Nacional de Ciencia y Tecnología (SNCT) y las Jornadas Estatales de Ciencia, Tecnología e Innovación (JECTI); curso de procuración de fondos, que se dio de forma gratuita; entregas de reconocimientos de eventos y la convocatoria del premio divulgador del año, la cual se trabajó por parte de todos los miembros durante las reuniones. Esta última se declaró desierta debido a que a su cierre únicamente se tuvieron dos propuestas; no se consideró pertinente darle avance.

En cuanto a los jueces considerados para evaluar, estaban Daniel de la Torre, Ernesto Márquez Nerey, Estrella Burgos, Miguel García y Antimio Cruz. Se propuso por primera vez el Premio al Divulgador del Año, mismo que desafortunadamente se declaró desierto.

Durante $2015,{ }^{8}$ se buscó ser más eficientes en el uso del tiempo y en las reuniones, por lo que se realizaron las siguientes actividades: cursos gratuitos para los miembros de la Red sobre procuración de fondos y creación de empresas de divulgación, cuatro reuniones a lo largo del año y el $2 .^{\circ}$ Encuentro de Divulgadores, en el cual se tuvo la participación de integrantes del Consejo de Guanajuato; los miembros de la Red, nuevamente apoyaron en las actividades del "Día del Niño" y se presentaron las convocatorias para participar en las actividades del proyecto de apropiación social en cuanto a la SNCT y las JECTI, el Domo, actividades con Zacatecas (Ciencia en Movimiento), Radio Curiosos (Querétaro) y la invitación para que asistieran a la Feria Nacional de Ciencias e Ingenierías 2015.

Como parte de la orden del día de las reuniones ordinarias, se presentaron las actividades de divulgación que realizan las IES y Cl miembros de la Red. En el mes de diciembre, se realizaron dos reuniones para desarrollar el Plan Estatal de Divulgación propuesto por la Red. Por segundo año consecutivo, se impulsó la propuesta del Premio al Divulgador del Año, mismo que volvió a no tener propuestas suficientes para ejecutarse, por lo que se consideró que no sería viable seguirlo impulsando.

Dos años después del ejercicio de planeación estratégica, se atendió el objetivo número tres por primera vez: diseñar indicadores de evaluación para medir y analizar el desempeño de la Red. A través de las $6^{\text {as }}$ Jornada Estatales de CTI, programa que consistió en visitar los 58 municipios del estado, mediante talleres de apropiación social del conocimiento. Este programa cambió de nombre a partir de 2016 y hasta 2018, con el nombre de Feria Municipal de Ciencia y Tecnología y en 2019 con el nombre de Semana Estatal de las Humanidades, Ciencias y Tecnologías. Sin embargo, a pesar del cambio de nombre, el programa mantiene la misma dinámica, que consiste en talleres de CTI a lo largo de los municipios de las cuatro regiones de San Luis Potosí (altiplano, centro, media y huasteca) durante una jornada escolar, tanto en horario matutino como vespertino.

Cabe destacar que, en los veinte años de existencia del Copocyt, jamás se había realizado una evaluación de los talleres. De acuerdo con declaraciones del Mtro. Hugo Alberto Jasso Villarreal, fundador y director general del Tife y responsable de divulgación del Copocyt en su fundación en 1996, no hay registro de evaluaciones previas de actividades de divulgación. Esto también ponía en tela de juicio la pertinencia de los temas que se habían llevado durante años a las regiones, asimismo, justificaba la asignación del recurso y el diseño más grande de logística en materia de talleres.

\footnotetext{
7 Copocyt, 2014. Reporte final del programa Apropiación social de la ciencia, tecnología e innovación San Luis Potosí 2014 "Ciencia para todos en todos los rincones"

8 Copocyt, 2015. Reporte final del programa Apropiación social de la ciencia, tecnología e innovación San Luis Potosí 2015 "Ciencia para todos".
} 
El recorrido comprendía la atención de los 58 municipios de los que está constituido el estado, incluyendo comunidades aparte de la cabecera municipal, dando atención de manera simultánea al objetivo 10 de la planeación estratégica (atender la totalidad de los municipios). Finalmente, el recorrido se realizó en 125 poblaciones en tan solo 4 meses, de agosto a noviembre de 2015, debido a que el programa con el cual se financiaba este proyecto (ENDDCTi, 2015) comprendía un periodo de ejecución máximo de 8 meses, y también existía dependencia del ciclo escolar vigente para que las escuelas fueran receptoras de la actividad; debía de ejecutarse de manera simultánea en distintas regiones.

Bajo un panorama tan grande de atención en poco tiempo, el Copocyt emitió una convocatoria para atención de las regiones, dividiendo el estado en 8 zonas (altiplano este y oeste, centro este y oeste, media este y oeste, y huasteca norte y sur), para que las instituciones miembros de la Red, presentaran propuestas, incluyendo el desglose de sus talleres y desarrollando una ficha técnica para cada uno. Cabe señalar que esto se enriqueció con el acercamiento a las direcciones municipales de educación de cada ayuntamiento en donde se les solicitaba acerca de cuáles eran los temas prioritarios de atención en la población.

Desafortunadamente, esto se hizo de manera informal a través de llamadas telefónicas y recabando información general, sin embargo, se rescataron los temas siguientes como prioritarios, esto a partir de lo sugerido por los municipios que participaron en las sugerencias: alimentación infantil, agua, generación de energía, calidad de vida, electricidad y vivienda. Se dieron seis meses (enero a junio de 2015) para que los proponentes desarrollaran sus propuestas. La convocatoria incluía, con detalle, cada una de las zonas, los periodos en los que se permitiría atender, la logística de protocolo, los códigos de vestimenta, así como reconocer, en todo momento, al Copocyt y al Conacyt por el evento.

Se presentaron diez instituciones, de las cuales, solamente seis cumplieron con los requisitos administrativos y técnicos solicitados. Respecto a lo administrativo, principalmente era cumplir con documentación para generar factura, comprobantes de domicilio e identificación del responsable legal. Cabe destacar que no todos cumplían con el requisito por no estar al corriente con el órgano tributario del estado, asimismo, existía irregularidad entre el domicilio fiscal en contraparte del domicilio legal.
Respecto a los requerimientos técnicos, consistía en indicar las actividades de manera específica, la zona de atención a la cual estaban interesados en atender, el desglose del currículo de los talleristas que conformaban el equipo de trabajo y el desglose financiero sobre el costo de materiales, traslados, alimentos, hospedajes y honorarios.

El ejercicio de evaluación se realizó durante el mes de julio, en el cual se tenían definidos a los grupos que atenderían cada una de las regiones. Cabe señalar que cada proponente debía de presentar sus respectivos talleres con recursos propios; y el Copocyt no asignaría el recurso hasta que estos fueran evaluados y validados por el comité de evaluación de los talleres.

Se desarrolló una rúbrica que contenía los siguientes elementos: pertinencia de la temática del taller, habilidad expositiva, capacidad de captar la atención del público, enfoque en la población objetivo, pertinencia de los materiales, capacidad de replicar el taller por los mismos usuarios, riesgo en el manejo de materiales y costo por taller.

De las seis instituciones evaluadas, dos tuvieron que realizar ajustes a sus presentaciones, incluso algunos tuvieron que cambiar de tallerista, dado que no tenían las habilidades expositivas que el comité consideraba para visitar poblaciones al interior del estado. Todas las evaluaciones se realizaron en el espacio de divulgación del copocyt, "El vagón de la ciencia", ubicado en uno de los principales parques de San Luis Potosí, el Parque Tangamanga. A este lugar se citaba a un cuerpo de al menos 5 evaluadores de instituciones distintas a las evaluadas, para que llenaran la rúbrica e hicieran de forma vivencial todos los talleres que se desarrollaron.

Es importante hacer mención que lo evaluado únicamente era el contenido de divulgación y la actividad como tal, sin embargo, algo que no se había considerado eran las capacidades administrativas, logísticas y de gestión de cada institución para hacer frente a las contingencias que se presentaron a lo largo del programa. Esto es, cómo enfrentar situaciones de desastres naturales como inundaciones que dejaban inaccesibles los poblados, cancelación de sedes, presencia de grupos del crimen organizado y cambios en los costos operativos. Esto dejó en evidencia que las habilidades, no solo de divulgación sino también de gestión, son un elemento fundamental en el desarrollo de divulgadores científicos. 
Ante el cambio de la Dirección General del Copocyt en 2016, ${ }^{9}$ las estrategias ordenadas cambiaron de rumbo en cierto sentido. Sin embargo, se logró mantener y darle continuidad al "Encuentro de Divulgadores Rediciti" en su tercera edición, evento que continuaba sin financiamiento, limitándose a la capacidad de gestión del Copocyt para obtener sedes y ponentes sin costo. Dentro de lo positivo, estuvo el impulso del primer cuaderno de ejercicios de matemáticas para niños de quinto grado de primaria, esto en colaboración con la Dirección de Educación Básica de la Secretaría de Educación de Gobierno del estado de San Luis Potosí. Este cuaderno fue realizado por especialistas de la Sociedad Mexicana de Matemáticas A.C. El cuaderno consistía en dos versiones, la impresa con doce ejercicios, y la versión digital, con veinte. Este cuaderno permitió realizar el segundo ejercicio de evaluación realizado por miembros de la Red del programa Divertimáticas, el cual consistía en convertir los ejercicios en una actividad lúdica para los alumnos sin que tuvieran que utilizar propiamente el cuaderno.

Este ejercicio de evaluación fue muy enriquecedor debido a que las sesiones de evaluación se hicieron directamente con alumnos de quinto grado de escuelas públicas, realizándoles encuestas sobre cuál había sido la actividad más entretenida, la que más les había dejado conocimiento y las cualidades expositivas del tallerista.

La evaluación de los talleres del programa Divertimáticas, permitió apoyar a cinco grupos de la Red, quienes fueron los proveedores del Copocyt en este programa, atendiendo un total de 138 escuelas primarias en el estado a través de talleres lúdicos e interactivos orientados a generar interés por las matemáticas. Algunos problemas generados debido a la mala planeación del programa, fueron: la retroalimentación entre pares generó fricción entre los mismos miembros que participaron como proveedores, no se generaron fichas detalladas de la dinámica de cada juego y solo quedaron en video.
Para 2017, ${ }^{10}$ y por instrucciones del Gobernador del estado, Dr. Juan Manuel Carreras López, se dio la indicación de continuar con los cuadernos de matemáticas. En esta ocasión para primero y tercero de secundaria, dado que, al cierre del proyecto de 2016, se logró consolidar el de segundo de secundaria. Sin embargo, los recursos financieros no permitieron desarrollar la estrategia de visitar las poblaciones al interior del estado con nuevas actividades en las escuelas; y se limitó a la impresión y distribución de los cuadernos en el sistema público educativo. En donde la Red continúo una participación intensa fue en el evento "Fiesta de las Ciencias y las Humanidades", impulsado por la Dirección General de Divulgación Científica (DGDC) de la Universidad Nacional Autónoma de México (Unam), siendo coordinador estatal el Copocyt en colaboración con la Rediciti. Asimismo, se realizaron 13 ferias municipales de ciencia y tecnología, esto a partir de la iniciativa creada en el mismo año de impulsar los Consejos Municipales de Ciencia y Tecnología, mejor conocidos como Comucyt.

Finalmente, en $2018,{ }^{11}$ bajo la directriz del director general, se impulsaron 27 ferias municipales de ciencia y tecnología en las cuatro regiones del estado, sin embargo, la dinámica no permitió realizar de forma sistemática evaluaciones ni encuestas de percepción de las actividades en dichas ferias. Esto se considera una oportunidad definitivamente desaprovechada para la obtención de datos cualitativos, dado que solo se tienen cuantitativos y la descripción de los talleres realizados en cada feria. Un aporte del programa de este año fue la continuidad de la "Fiesta de las Ciencias y las Humanidades", de la DGDC de la Unam, en donde se abrió un escaparate para que los divulgadores de la Red desarrollaran competencias como conferencistas y no como talleristas, lo que les permitió desde entonces desarrollarse no solamente en talleres expositivos, sino en conferencias para distintos públicos. En 2018 se realizó la quinta edición del "Encuentro de Divulgadores Rediciti", con la participación de conferencistas foráneos y comunicadores de renombre de medios de comunicación masivos, asimismo, se incluyó la técnica de stand up y divulgación con enfoque de género.

9 Copocyt, 2016. Reporte final del programa Apropiación social de la ciencia, tecnología e innovación San Luis Potosí 2016.

10 Copocyt, 2017. Reporte final del programa Apropiación social de la ciencia, tecnología e innovación San Luis Potosí 2017.

11 COPOCYT, 2018. Reporte final del programa Apropiación social de la ciencia, tecnología e innovación San Luis Potosí 2018. 


\section{Resultados}

Recapitulando los diez objetivos planteados, y en un esfuerzo por alinearlos con los principios de lo que se espera de lo que sea una sociedad del conocimiento, junto con los trabajos realizados con los proyectos del Conacyt en San Luis Potosí. La consolidación se dará en función a su capacidad de adaptarse a las necesidades de comunicación pública del conocimiento científico, tecnológico y de innovación, este objetivo deberá de ser una directriz activa mientras exista la Red. Las capacitaciones desde 2012 y hasta 2016, junto con los encuentros de divulgadores, buscaron la integración y profesionalización, sin embargo, no son claros los objetivos y la forma de seguimiento de sus integrantes, más allá de su registro anual que deban de actualizar.

Las actividades que se consolidaron de forma exitosa son las siguientes:

1. Base de registros a partir de 2013.

2. Asignación de recursos económicos para las 4.a, 5.a y 6.a Jornadas Estatales de Ciencia, Tecnología e Innovación.

3. Asignación de recursos económicos para las 20.a, 21.a y 22.a Semana Nacional de Ciencia y Tecnología.

4. Apoyo sin asignación de recursos para la celebración del "Día del Niño" en 2014, 2015 y 2016.

5. Asignación de recursos a través de cuatro convocatorias del proyecto Apropiación Social de la CTI en 2015: "Divulgadores de Oro" (capacitación de adulto mayor como divulgador en el estado de Aguascalientes), "Domo Curioso" (planetario inflable a las regiones), "Radio Curiosos" (cápsulas radiofónicas para Querétaro), "Ciencia en Movimiento" (talleres de divulgación en Zacatecas y San Luis Potosí). En 2016, "Divertimáticas", "El Jardín del Desierto" y el "Domo Curioso", los tres propiedad del Copocyt.

6. Creación del catálogo de instituciones de la Rediciti en 2016.

Por el contrario, las principales propuestas que durante el periodo de la implementación no se consolidaron fueron las siguientes:

1. Creación de la oficina de la Red a través del donativo de infraestructura y capital de los mismos miembros.

2. Realización del Anuario 2013.

3. Creación de un Consejo Directivo en 2013.
4. Página web en 2013.

5. Acta Constitutiva en 2013.

6. Creación de Comités de Trabajo en 2014.

Asimismo, se reunieron las principales recomendaciones realizadas por los miembros de la Red a su administrador, las cuales se concentran en los siguientes puntos:

1. Para cuestiones de diseño, se deberá convocar a todas las instituciones miembros que cuenten con área de diseño gráfico.

2. Evaluar la pertinencia de miembros que no sean de San Luis Potosí.

3. Generar un comité organizador para el Premio Divulgador del Año.

4. Generar un comité organizador para el "Encuentro de Divulgadores".

5. Derechos de miembros invitados a la Red.

6. Delegación de actividades, construcción de comisiones o grupos de trabajo.

Resumen de actividades realizadas en el periodo que se indica de mayo de 2013 a 2019

1. Años de operación: 9 (2010 a 2019).

2. Reuniones ordinarias: 29 (2013 a 2019, no se cuenta con registros de los tres primeros años).

3. Reuniones extraordinarias: 1 (marzo 2013).

4. Cursos gratuitos a los miembros: 5 (impartidos por los mismos miembros de la Red y especialistas invitados, quienes no cobraban honorarios).

5. Encuentros de divulgadores: 5 (tres realizados a través de gestiones y apoyos interinstitucionales y 2 financiados por Conacyt)

6. Instituciones presentadas durante las reuniones: 23 .

Desde 2016 a la fecha, la base de datos de la Red ha servido como directorio de enlaces de difusión y divulgación en donde se difunden convocatorias de apoyo, principalmente del Conacyt, tales como becas, proyectos, etc. Igualmente cuenta con Facebook, en donde se publican actividades propias de la Red y temas de su interés.

A pesar de los esfuerzos de desarrollar un plan estatal de divulgación en 2015, es hasta 2019 cuando se espera que se realice dicho ejercicio con miras de establecerlo para 2027 cuando terminaría la siguiente administración estatal. 
Como se mencionó en apartados anteriores, solamente se han realizado dos ejercicios de evaluación. Sin embargo, no se puede decir que ya existe de forma sistematizada una estructura de indicadores cualitativos y cuantitativos para divulgación, únicamente aquellos que se manejan como parte de los reportes finales de los proyectos, tales como número de personas atendidas, número de actividades realizadas, número de municipios visitados y número de entidades federativas en colaboración.

Respecto a generar un mecanismo de sustentabilidad financiera, es hasta 2017 cuando destella una luz de oportunidad en la que los grupos de divulgación ChipOhm y Science Coaching fueron contratados de forma directa por una empresa para realizar una semana de actividades de divulgación con estudiantes de primaria. Sin embargo, siguen siendo muy aislados y poco vinculados los esfuerzos de generar recursos aparte de los que el Copocyt asigna a través de los apoyos federales.

Así como el objetivo número uno sobre consolidación de la Red, el realizar actividades de divulgación en ámbitos educativos mediante la creación de talleres innovadores que permitan despertar el interés deberá de ser una tarea permanente. A partir de la reforma educativa de la Secretaría de Educación Pública de México, aparecen los clubes de actividades, los cuales consisten en orientar actividades específicas con los alumnos para que desarrollen ciertas competencias humanas y destrezas en áreas de ciencias duras. Esto abre una posibilidad de adentrar la divulgación como parte de las actividades programadas en el calendario escolar, e incluso fomentar un sistema de autosustentabilidad financiera en un mediano y largo plazo.

Si bien ya se mencionó acerca de la capacitación y formación de divulgadores, sigue siendo muy escasa la participación de grupos e instituciones para la divulgación al interior del estado, generando altos costos operativos para llevar las actividades. Apenas en 2018, las ferias municipales de ciencia y tecnología, se sumaron los institutos tecnológicos de Tamazunchale, Superior de Ébano (ambos región huasteca) y Rioverde (región media) lideraron las actividades en su propio municipio. Sin embargo, faltan muchas alianzas por desarrollar.

A la par de que los miembros de la Red generen sus propios recursos, existe aún fuerte dependencia de las convocatorias y apoyos públicos. Sigue sin explotarse la posibilidad de que la iniciativa privada, a través de las empresas manufactureras y de servicio, inviertan en la divulgación no por falta de interés, sino por falta de acercamiento de los grupos e instituciones hacia las pequeñas, medianas y grandes empresas de la zona.

Respecto al objetivo de triplicar el número de divulgadores en el estado, actualmente la Red cuenta con 198 miembros de 62 instituciones registradas, integradas en 9 entidades pertenecientes a universidades públicas, 3 institutos tecnológicos, una institución de nivel medio superior, 3 centros de investigación, 9 instituciones de gobierno del estado, 4 instituciones educativas privadas y 33 instituciones dedicadas a la divulgación privada. Sin embargo, el $90 \%$ se sigue concentrando en la capital y zona metropolitana, dejando aún desprotegido las poblaciones al interior del estado. El hecho de que se tenga un crecimiento exponencial, no indica que todas las personas participen activamente en todos los eventos, dado que existen miembros que solamente se registran ante la Red por cuestión curricular y no por participar en actividades de gestión o de campo.

El objetivo de atender la totalidad del estado se cumplió en 2015, de forma presencial y de 2014 a 2017 de forma virtual, a través de la entrega de los cuadernos de "Divertimáticas". Sin embargo, es de considerar que haber visitado los 58 municipios no es indicativo de atender a la totalidad de la población de cada ayuntamiento, debido a que las actividades principalmente se desarrollaron en las cabeceras de cada municipio. Por lo anterior, es fundamental la creación de instituciones in situ para la continuidad de las actividades.

Los pilares que sustentan el concepto de sociedad del conocimiento solo se podrán analizar bajo un seguimiento de al menos 5 años a estudiantes tanto de primaria como de secundaria, realizando encuestas y estudios de historias de vida con la finalidad de saber si las actividades propuestas de divulgación fueron el factor detonante de la vocación científica y no aquel profesor o profesora de biología, química, física, matemáticas o historia que, ante su pasión por enseñar, estimuló al estudiante para convertirse en científico.

\section{Conclusiones}

El que no sean evaluadas las actividades de divulgación, tanto cualitativa, como cuantitativamente, podrá generar "ceguera de taller" para la Red, tal como el Encuentro de Divulgadores, o realizar talleres de ciencia y tecnología 
en las distintas poblaciones que conforman el estado, sin realizar estudios de percepción pública de las humanidades, ciencias, tecnología e innovación, tanto antes como después de la ejecución de las actividades. Solo el seguimiento y el análisis de información, como testimoniales de los asistentes a las actividades, permitirán una Red consolidada en sus objetivos principales, visión y misión. Asimismo, la evaluación del porqué de la pertenencia de cada miembro; si es solamente, como se dice coloquialmente, "para salir en la foto", o es para promover el juicio crítico en la población.

Distinto a la publicación de artículos en revistas indexadas, ponencias o participación en congresos, la divulgación debe de documentarse en toda su estructura, esto es, desde el desarrollo de las fichas técnicas de talleres hasta la fotografía, grabación y el registro de testimoniales de los usuarios para validar la existencia de actividades de divulgación. Hay que considerar que la expedición de un sencillo diploma, una constancia o un reconocimiento de participación no habla de la calidad de las actividades o de su impacto.

Con el objetivo de que la estructura de la Red tenga respaldo institucional, es fundamental que todo sea validado por el Honorable Consejo Directivo del Copocyt, órgano rector del sector de ciencia y tecnología ante el Gobierno del estado.

Es fundamental darle continuidad a la generación del catálogo de miembros de la Rediciti, con el objetivo de promoverlo y distribuirlo a los tomadores de decisión de actividades de divulgación de las HCT en todo el país.

Es básico rescatar que la labor del Nuevo Consejo Nacional de Ciencia y Tecnología de la República de El Salvador, a través de las gestiones de la Licda. Elba Membreño, responsable de popularización de la ciencia y tecnología, ha permitido la apertura de articulación internacional entre la Rediciti y El Salvador para el intercambio de actividades, divulgadores e información. Deberá ser una actividad sustantiva en el proceso de vinculación y consolidación de la Red.

La cobertura constante en el estado, solamente se podrá dar a partir del impulso, creación y apoyo de grupos al interior del estado, aunado a un elemento indispensable de promover la industria de la divulgación, esto es, no mercantilizarla, sino hacer que la iniciativa privada apoye en programas tanto en la capital, como al interior del estado, para incentivar un entorno de apropiación social del conocimiento.
Continuar bajo lineamientos de normativa administrativa de asignar todo recurso público a través de convocatorias públicas sin importar el monto de apoyo para la ejecución de actividades de divulgación.

El proceso de formación de divulgadores, deberá de incluirse el desarrollo y evaluación de actividades y productos dirigidos a personas con discapacidad y grupos subrepresentados como comunidades indígenas.

Invitar e impulsar que los miembros líderes gestionen la formalización de la Red a fin de que puedan definir su propia identidad y el plan de acción para cada año, junto con la posibilidad de estar facultados en participar en convocatorias del Conacyt o de alguna otra índole de forma independiente al Copocyt. Gestionar recursos y posicionarse como una institución especializada en la comunicación pública del conocimiento científico y tecnológico.

Se recomienda que sea creado el grupo de consejeros líderes de la Rediciti, conformado por siete representantes, uno por cada tipo de institución, todos miembros activos, recomendando que sea de la siguiente forma:

1. Universidad pública

2. Instituto tecnológico

3. Institución de media superior o bachillerato

4. Centro de investigación

5. Gobierno estatal

6. Educación privada

7. Grupos de divulgación

Este grupo permitirá ser órgano consultor y asesor, que represente a todos los miembros de forma equitativa, e incidir en la política pública para mejores prácticas de la divulgación en la transición hacia la sociedad del conocimiento en San Luis Potosí.

\section{Referencias}

Consejo Nacional de Población. (2016). Índice de marginación por entidad federativa y municipio 2015. Recuperado de https://www.gob.mx/conapo/documentos/indice-demarginacion-por-entidad-federativa-y-municipio-2015

COPOCYT. (2013). Reporte final del programa "Apropiación social de la ciencia, tecnología e innovación con énfasis en zonas marginadas del estado de San Luis Potosí 
2013". San Luis Potosí, México: Consejo Potosino de Ciencia y Tecnología

COPOCYT. (2014). Reporte final del programa "Apropiación social de la ciencia, tecnología e innovación San Luis Potosí 2014". San Luis Potosí, México: Consejo Potosino de Ciencia y Tecnología

COPOCYT. (2015). Reporte final del programa "Apropiación social de la ciencia, tecnología e innovación, San Luis Potosí 2015". San Luis Potosí, México: Consejo Potosino de Ciencia y Tecnología

COPOCYT. (2016). Reporte final del programa "Apropiación social de la ciencia, tecnología e innovación, San Luis Potosí 2016". San Luis Potosí, México: Consejo Potosino de Ciencia y Tecnología

COPOCYT. (2017). Reporte final del programa "Apropiación social de la ciencia, tecnología e innovación, San Luis Potosí 2017". San Luis Potosí, México: Consejo Potosino de Ciencia y Tecnología

COPOCYT. (2018). Reporte final del programa "Apropiación social de la ciencia, tecnología e innovación, San Luis Potosí 2018". San Luis Potosí, México: Consejo Potosino de Ciencia y Tecnología

Gobierno de México. (2013). Plan Nacional de Desarrollo 2013-2018 [versión de Adobe Acrobat Reader].
Recuperado de https://www.snieg.mx/contenidos/ espanol/normatividad/MarcoJuridico/PND_20132018.pdf

Gobierno del Estado de San Luis Potosí. (2015). Plan Estatal de Desarrollo 2015-2021, San Luis Potosí, México. Recuperado de http://www.slp.gob.mx/plan2015-2021/

Krüger, K. (octubre, 2006). El concepto de sociedad del conocimiento. Revista bibliográfica de geografía y ciencias sociales, 11(683). Recuperado de http://www. ub.edu/geocrit/b3w-683.htm

Padilla, J. y Patiño, L. (2016). Fortalecimiento de la apropiación social de la ciencia y la tecnología en los estados en 2012. Modelo conceptual sobre apropiación social, cultura y alfabetismo científico. Ciudad de México, México: Red Nacional de Consejos y Organismos Estatales de Ciencia y Tecnología.

UASLP. (2017). México debe caminar hacia la sociedad del conocimiento: Enrique Cabrero. Recuperado http://www.uasIp.mx/Comunicacion-Social/Paginas/ Divulgacion/Gaceta/Notas/ 2017/Octubre/061017/ Enrique-Cabrero-conferencia.aspx

UNESCO. (2016). Construir sociedades del conocimiento. Recuperado de https://es.unesco.org/ themes/ construir-sociedades-del-conocimiento 